\title{
A SHARP FORM OF THE VIRIAL THEOREM ${ }^{1}$
}

BY HARRY POLLARD

\author{
Communicated by Maurice Heins, April 8, 1964
}

In its classical form the Virial Theorem concerns the behavior of a system $S$ of $n$ mass particles acting under Newtonian attraction in such a fashion that the center of mass 0 remains fixed and the potential energy $V$ satisfies $V>-\infty$ for all positive time $t$. The latter condition, which is not always stated explicitly, guarantees the analyticity of the coordinates of the particles in the independent variable $t$; in particular, it excludes collisions [2, pp. $324 \mathrm{ff}$.]. Let $T$ denote the kinetic energy and $h$ the (constant) total energy $T+V$. Let $\hat{V}$ denote the time average

$$
\hat{V}=\lim _{t \rightarrow \infty} \frac{1}{t} \int_{0}^{t} V(\tau) d \tau
$$

if the limit exists, with an analogous definition of $\hat{T}$. Clearly each of $\hat{V}, \hat{T}$ exists if the other does and $\hat{T}+\hat{V}=h$. The usual theorem states that if $S$ is bounded, in the sense that distances between particles and the velocities of the particles remain bounded, then $\hat{T}$ and $\hat{V}$ exist and $2 \hat{T}=-\hat{V}$. An equivalent conclusion is

$$
\hat{T}=-h \text {. }
$$

In this form the theorem is mathematically unsatisfactory because the condition of boundedness is far from necessary. This is already demonstrated by the parabolic case $h=0$ of the two-body problem, $n=2$. In this case distance grows like $t^{2 / 3}$, so that $V$ behaves like $-t^{-2 / 3}$ as $t \rightarrow \infty$. Consequently, $\hat{V}=0$. Hence $\hat{T}=0$, which is consistent with (1).

We shall replace boundedness by a condition which is both necessary and sufficient. Let $r_{j k}(t)$ denote the distance between particle $j$ and particle $k$ at time $t$, and let $R(t)=\max _{j, k} r_{j k}(t)$.

THEOREM 1. (1) is true if and only if

$$
R(t)=o(t), \quad t \rightarrow \infty .
$$

Let $2 I$ denote the moment of inertia of the system with respect to 0 . We begin by showing that (2) is equivalent to

${ }^{1}$ Presented at the Summer Institute for Dynamical Astronomy, Yale University, July 24, 1962. 


$$
I(t)=o\left(t^{2}\right), \quad t \rightarrow \infty .
$$

Since $[2$, p. 243]

$$
2 I M=\sum_{1 ฐ j<k \leqq n} m_{j} m_{k} \underset{r_{j k}}{2},
$$

where $M$ is the total mass, it follows from the definition of $R$ that $I<C_{1} R^{2}$, where $C_{1}$ is independent of time. On the other hand, according to (4),

$$
2 I M>m m^{\prime} \sum \underset{r_{j k}^{2}}{2}
$$

where $m, m^{\prime}$ are the two smallest masses. At each instant of time $R(t)$ is one of the $r_{j k}$. Therefore, $2 I M>m m^{\prime} R^{2}$, so $C_{2} R^{2}<I$ where $C_{2}$ is a positive constant independent of time. Hence

$$
C_{2} R^{2}<I<C_{1} R^{2},
$$

which implies the equivalence of (2) and (3).

From an integration it is clear that the relation

$$
\ddot{I}(t)=o(t), \quad t \rightarrow \infty,
$$

implies (3). That it is implied by (3) is more subtle. According to the Lagrange identity $[2$, p. 235],

$$
I=T+h .
$$

Since $T \geqq 0, \ddot{I} \geqq h>-\infty$. By a theorem of Landau (see, for example $\left[1\right.$, Theorem $1 \mathrm{~B}_{2}$, p. 638$]$ ) this property of $\ddot{I}$ entitles us to differentiate each side of (3) to obtain (5). We have proved the equivalence of (2), (3), (5).

Now integrate both sides of (6) and divide by $t$. Then

$$
\frac{I(t)}{t}=\frac{1}{t} \int_{0}^{t} T(\tau) d \tau+h+o\left(\frac{1}{t}\right), \quad t \rightarrow \infty .
$$

It follows that (5) and (1) are equivalent. Hence so are (1) and (2). The theorem stands established.

The next theorem holds with no a priori assumptions on the growth of $S$.

THEOREM 2. We have

$$
\limsup _{t \rightarrow \infty} \frac{1}{t} \int_{0}^{t} T(\tau) d \tau \geqq|h| .
$$

Denote the left-hand side by $L$. Since $T=-V+h$ and $V \leqq 0$ it follows that $T \geqq h$. Hence $L \geqq h$. On the other hand, according to ( 7 ) 


$$
\limsup _{t \rightarrow \infty} \frac{\dot{I}(t)}{t}=L+h
$$

I claim $L+h \geqq 0$; otherwise $\dot{I}(t) \leqq-\epsilon t$ for large $t$, where $\epsilon>0$. Integrating and dividing by $t^{2}$ yields

$$
\limsup _{t \rightarrow \infty} \frac{I(t)}{t^{2}} \leqq-\epsilon,
$$

which is impossible since $I \geqq 0$. Hence $L \geqq h, L \geqq-h$, from which the theorem follows.

Corollary. If $\hat{T}=0$, then $h=0$ and $R(t)=o(t)$.

The first conclusion follows from Theorem 2. Then the second follows from Theorem 1 .

I am indebted to Professor M. Golomb for observing that my original proof of the Corollary actually proves the stronger Theorem 2.

\section{REFERENCES}

1. R. P. Boas, Asymptotic relations for derivatives, Duke Math. J. 3 (1937), 637646.

2. A. Wintner, The analytical foundations of celestial mechanics, Princeton Univ. Press, Princeton, N. J., 1942.

Purdue University 